\title{
The atrophy of white and gray matter volume in patients with comitant strabismus: Evidence from a voxel-based morphometry study
}

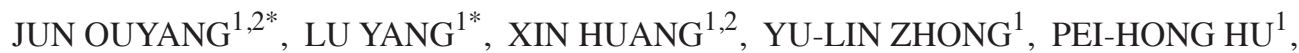 \\ YING ZHANG $^{1}$, CHONG-GANG PEI ${ }^{1}$ and YI SHAO $^{1}$ \\ ${ }^{1}$ Department of Ophthalmology, The First Affiliated Hospital of Nanchang University, \\ Jiangxi Province Clinical Ophthalmology Institute and Oculopathy Research Centre, Nanchang, Jiangxi 330006; \\ ${ }^{2}$ Department of Ophthalmology, The First People's Hospital of Jiujiang, Jiujiang, Jiangxi 332000, P.R. China
}

Received August 16, 2016; Accepted May 11, 2017

DOI: $10.3892 / \mathrm{mmr} .2017 .7006$

\begin{abstract}
To compare the difference in white matter volume (WMV) and gray matter volume (GMV) between the comitant strabismus (CS) patients and health controls by voxel-based morphometry (VBM) and the relationship with behavioral performance. A total of 20 patients with comitant strabismus (10 males and 10 females), and 20 healthy subjects (10 males and 10 females) with matched age, sex status underwent magnetic resonance examination. The authors analyzed the original 3D T1 brain images using the VBM module. The comitant strabismus groups were compared with the control groups for the GMW and WMV of the entire brain. Correlation analysis was performed to investigate the relationship between the GMV and WMV altered areas and the behavioral performance in comitant strabismus. CS patients were distinguishable from the healthy controls (HCs) by receiver operating characteristic curves. Results of the VBM analysis demonstrated that the CS groups had decreased GMV in the brain regions of the left middle temporal pole, left cerebellum posterior lobe, right posterior cingulate cortex, left cuneus and right premotor cortex. Meanwhile, the WMV was significantly decreased in the brain regions of the left middle temporal gyrus, right middle temporal gyrus, left middle temporal gyrus, right precuneus and right premotor cortex in the comitant strabismus patients compared with HCs. Furthermore, the duration of CS was negatively correlated with the GMV values of the left
\end{abstract}

Correspondence to: Dr Yi Shao, Department of Ophthalmology, The First Affiliated Hospital of Nanchang University, Jiangxi Province Clinical Ophthalmology Institute and Oculopathy Research Centre, 17 YongWaiZheng Street, DongHu, Nanchang, Jiangxi 330006, P.R. China

E-mail: freebee99@163.com

*Contributed equally

Key words: comitant strabismus, voxel-based morphometry, gray matter volume, white gray volume middle temporal pole ( $r=-0.486, \mathrm{P}=0.030)$. $\mathrm{CS}$ caused GMV and WMV atrophy in many brain regions, which may indicate the neural mechanisms of the ocular motility disorders in CS patients.

\section{Introduction}

Comitant strabismus (CS) is a common form of strabismus characterized ocular motility disorders, which may lead to compromised binocular vision and amblyopia $(1,2)$. The birth occurrence of infantile esotropia is $\sim 25$ per 10,000 in the United States (3). Strabismus is characterized by a constant angle deviation in many directions of the gaze. Clinically, CS is also associated with stereopsis impairment (4).

Previous research revealed the abnormal brain function in strabismus patients. A study exhibited that there had reduced functional connectivity between the V1 and V2 areas in monkeys with strabismic amblyopia (5). Another study reported that there is increased mean diffusivity of the occipital tracts in patients with strabismic amblyopia (6). Additionally, a previous study demonstrated that patients with strabismus were associated with suppression in the primary visual cortex (7). Although the abovementioned studies have demonstrated functional changes in the neurons of patients with strabismus, the changes in brain anatomical morphology in $\mathrm{CS}$ remain unknown.

Voxel-based morphometry (VBM) is a whole-brain measurement method that compares voxel-wise between groups morphological differences in the brain (8). The VBM method has been successfully used to assess neural mechanisms of nervous and mental diseases such as Alzheimer's disease (9), optic neuritis (10) and schizophrenia (11). Using the VBM method, a previous study indicated that the lower GMV values were located in the brain regions of the occipital eye field and parietal eye field in patients with strabismus amblyopia (12). In the present study, the authors demonstrated that there were many brain regions with a dysfunction in neural activity in patients with CS using regional homogeneity methods. However, there were far less evidence for neuromorphological changes. To the best of the authors' knowledge, the 
current study is the first to use the VBM approach to detect changes in volumes of the GMV and WMV of CS patients.

\section{Materials and methods}

Subjects. A total of 20 patients with CS (10 males and 10 females) were recruited from the First Affiliated Hospital of Nanchang University Hospital (Nanchang, China). The criteria of the study on CS were as follows: i) Strabismus at birth; ii) strabismus with impairment to stereopsis and visual fusion; iii) equal binocular best corrected vision; iv) the a range of squint angle is 50-60 delta.

Patients with following conditions were excluded from the study: i) Acquired strabismus, incomitant strabismus; ii) conditions due to eye diseases (infection, inflammation, ischemic diseases), iii) patients with eye surgery; iv) psychiatric disorders cardiovascular diseases, cerebral infarction diseases such as systemic disorders; v) alcohol or drug addiction.

A total of $20 \mathrm{HCs}$ (10 males and 10 females) with matched age, sex, education status were also recruited for the study. All HCs met the following criteria: i) No abnormalities in brain parenchyma with head magnetic resonance imaging (MRI); ii) without any eye diseases and the corrected visual acuity (VA) $>1.0$; iii) no nervous system diseases; iv) no external accessories that interfere with the magnetic resonance imaging signal (such as cardiac pacemaker or metal device).

All the research contents and methods followed the Declaration of Helsinki. All volunteers were informed of the purposes, methods and potential risks before signing an informed consent form. The study was approved by the medical ethics committee of the First Affiliated Hospital of Nanchang University Hospital (Nanchang, China).

MRI parameters. All subjects were performed with a 3-Tesla MR scanner (Siemens AG, Munich, Germany) with an 8-channel. High-resolution T1-weighted images were obtained with a magnetization-prepared rapid gradient echo (MP-RAGE) sequence. The details of scanning parameters are as follows: Slices $=176$; section thickness $=1.0 \mathrm{~mm}$; echo time $=2.26 \mathrm{msec}$; repetition time $=1,900 \mathrm{msec}$; field of view $=215 \times 230 \mathrm{~mm}$.

VBM analysis. Structural images were classified by MRIcro software (version, 1.40; build, 1; www.MRIcro.com) to eliminate incomplete data, and then processed with the voxel-based morphometry toolbox (VBM8) (dbm.neuro.uni-jena.de/vbm8) implemented in Statistical Parametric Mapping software (version, 8.0; Wellcome Trust Centre for Neuroimaging,London, UK). All procedures were performed with MATLAB (version, 7.9.0; The Mathworks, Inc. Natick, MA, USA). Individual brain images was segregated into gray matter, white matter and cerebrospinal fluid based on the VBM8 toolbox. More details are presented in a previous study of the authors (10).

Statistical analysis. General linear model analysis was performed with the SPM8 toolkit to investigate the group differences in GMV and WMV between CS groups and HCs. $\mathrm{P}<0.05$ was considered to indicate a statistically significant difference. Voxel threshold was set to 20 neighboring voxels to analysis the area of gray matter changes in CS.
Brain-behavior correlation analysis. With the VBM findings, different brain regions were classified as regions of interests (ROIs) using REST software (version, 1.8; www.resting-fmri .Sourceforge.net). For each ROI, the mean GMV or WMV value was extracted by averaging the GMV or WMV values over all voxels. Finally, the relationship between the mean GMV value in different brain regions in the CS group and the clinical manifestations were investigated using correlation analysis. $\mathrm{P}<0.05$ was considered to indicate a statistically significant difference.

Clinical data analysis. All clinical data of the CS patients were collected, including the onset of CS disease, and bestcorrected VA.

\section{Results}

General data analysis. Compared with HCs and strabismus groups, the authors did not find marked differences in weight $(\mathrm{P}=0.918)$, age $(\mathrm{P}=0.344)$, best-corrected VA-Right $(\mathrm{P}=0.814)$ and best-corrected VA-Left $(\mathrm{P}=0.903$; Table I).

Gray and white matter differences. Compared with HCs, CS groups had significantly lower GMV in the brain regions of the left middle temporal pole, left cerebellum posterior lobe, right posterior cingulate cortex, left cuneus and right premotor cortex (Fig. 1 and Table II). Additionally, CS patients had significantly lower WMV in the brain regions of the left middle temporal gyrus, right middle temporal gyrus, right precuneus and right premotor cortex (Fig. 2 and Table III). Moreover, the authors demonstrated the mean of altered GMV and WMV between the two groups (Fig. 3).

Correlation analysis. In the CS groups, it was observed that the duration of comitant strabismus negatively correlated with the GMV values of the left middle temporal pole $(r=-0.486$, $\mathrm{P}=0.030$; Fig. 4).

Receiver operating characteristic (ROC) curve. The authors assumed that the differences of the WMV and GMV values in two groups may be useful diagnostic markers. The mean values of the WMV and GMV in different brain regions were extracted and used to analyze ROC curves. The areas under the ROC for GMV values were: The left middle temporal pole (0.795), the left cerebellum posterior lobe (0.835), the right posterior cingulate cortex (0.790), the left cuneus $(0.870)$ and the right premotor cortex $(0.830 ;$ Fig. $5 \mathrm{~A})$. The AUCs for WMV values were: The left middle temporal gyrus $1(0.810)$, the right middle temporal gyrus (0.818), the left middle temporal gyrus 2 (0.823), the right precuneus $(0.800)$ and the right premotor cortex (0.835; Fig. 5B).

\section{Discussion}

To the best of the authors' knowledge, the current study is the first to evaluate changes in WMV and GMV in patients with CS using a VBM approach. They identified a remarkable decrease in GMV values in the brain regions of the left middle temporal pole, left cerebellum posterior lobe, right posterior cingulate cortex, left cuneus and right premotor cortex in patients with 
Table I. Demographic information and clinical measurements for CS and HCs.

\begin{tabular}{|c|c|c|c|c|}
\hline & CS & $\mathrm{HCs}$ & $\mathrm{t}$-value & P-values \\
\hline Male/Female & $10 / 10$ & $10 / 10$ & $\mathrm{~N} / \mathrm{A}$ & $>0.99$ \\
\hline Age (years) & $30.40 \pm 10.44$ & $27.45 \pm 8.99$ & 0.957 & 0.344 \\
\hline Weight (kg) & $60.25 \pm 6.46$ & $60.05 \pm 5.77$ & 0.103 & 0.918 \\
\hline Handedness (n, hand) & 20, right & 20, right & N/A & $>0.99$ \\
\hline Exotropic and esotropic (n) & $5 / 15$ & N/A & N/A & N/A \\
\hline Duration of strabismus (years) & $26.95 \pm 9.05$ & $\mathrm{~N} / \mathrm{A}$ & $\mathrm{N} / \mathrm{A}$ & $\mathrm{N} / \mathrm{A}$ \\
\hline Best-corrected VA-right & $1.07 \pm 0.18$ & $1.09 \pm 0.22$ & -0.237 & 0.814 \\
\hline Best-corrected VA-left & $1.02 \pm 0.09$ & $1.03 \pm 0.16$ & -0.123 & 0.903 \\
\hline
\end{tabular}

P-values, independent t-test. P-value between CS and HCs. CS, comitant strabismus; HCs, healthy controls; N/A, not applicable; VA, visual acuity.

Table II. Brain regions with significant differences in GMV between CS group and HCs.

\begin{tabular}{|c|c|c|c|c|c|c|c|}
\hline \multirow[b]{2}{*}{ GMV } & \multicolumn{4}{|c|}{ CS group and healthy controls } & \multicolumn{3}{|c|}{ MNI coordinates } \\
\hline & Brain areas & BA & Peak & lues & $\mathrm{x}$ & $\mathrm{y}$ & $\mathrm{z}$ \\
\hline \multicolumn{8}{|l|}{$\mathrm{CS}<\mathrm{HC}$} \\
\hline 1. & Left middle temporal pole & 21,38 & -4.179 & 1,077 & -33 & 12 & -37.5 \\
\hline 2. & Left cerebellum posterior lobe & - & -4.204 & 1,461 & -4.5 & -76.5 & -18 \\
\hline 3. & Right posterior cingulate cortex & 18,30 & -3.692 & 342 & 16.5 & -72 & 15 \\
\hline 4. & Left cuneus & $17,18,19$ & -3.871 & 1,034 & -13.5 & -78 & 6 \\
\hline 5. & Right premotor cortex & 6 & -3.752 & 348 & 3 & -42 & 48 \\
\hline
\end{tabular}

The statistical threshold was set at the voxel with $\mathrm{P}<0.05$ for multiple comparisons using Gaussian Random Field theory (voxels-wise $\mathrm{P}<0.01$ and cluster-wise $\mathrm{P}<0.05$ corrected). GMV, grey matter volume; CS, comitant strabismus; HCs, health controls, BA, Brodmann area; MNI, Montreal Neurological Institute; 1 voxel=1.5x1.5x1.5 mm.

Table III. Brain regions with significant differences in WMV between CS group and HCs.

\begin{tabular}{|c|c|c|c|c|c|c|c|}
\hline \multirow[b]{2}{*}{ WMV } & \multicolumn{4}{|c|}{ CS group and healthy controls } & \multicolumn{3}{|c|}{ MNI coordinates } \\
\hline & Brain areas & $\mathrm{BA}$ & Peal & lues & $\mathrm{x}$ & $\mathrm{y}$ & $\mathrm{z}$ \\
\hline \multicolumn{8}{|c|}{$\mathrm{CS}<\mathrm{HC}$} \\
\hline 1. & Left middle temporal gyrus 1 & 21,38 & -3.569 & 657 & -43.5 & 4.5 & -25.5 \\
\hline 2. & Right middle temporal gyrus & 39 & -4.345 & 424 & 42 & -52.5 & 7.5 \\
\hline 3. & Left middle temporal gyrus 2 & 18 & -3.420 & 222 & -7.5 & -90 & 12 \\
\hline 4. & Right precuneus & 7 & -3.871 & 1,034 & -13.5 & -78 & 6 \\
\hline 5. & Right premotor cortex & 6 & -3.752 & 348 & 3 & -42 & 48 \\
\hline
\end{tabular}

The statistical threshold was set at the voxel with $\mathrm{P}<0.05$ for multiple comparisons using Gaussian Random Field theory (voxels-wise $\mathrm{P}<0.01$ and cluster-wise $\mathrm{P}<0.05$ corrected). WMV, white matter volume; CS, comitant strabismus; HCs, healthy controls, BA, Brodmann area; MNI, Montreal Neurological Institute; 1 voxel $=1.5 \times 1.5 \times 1.5 \mathrm{~mm}$.

CS. Meanwhile, WMV values in the brain regions of the left middle temporal gyrus, right middle temporal gyrus, right precuneus and right premotor cortex were also significantly reduced in CS patients. Furthermore, the authors observed that the duration of comitant strabismus demonstrated a negative correlation with the GMV values of the left middle temporal pole.

The middle temporal gyrus is located between the superior temporal gyrus and inferior temporal gyrus, a region responsible for three-dimensional surface orientation and 


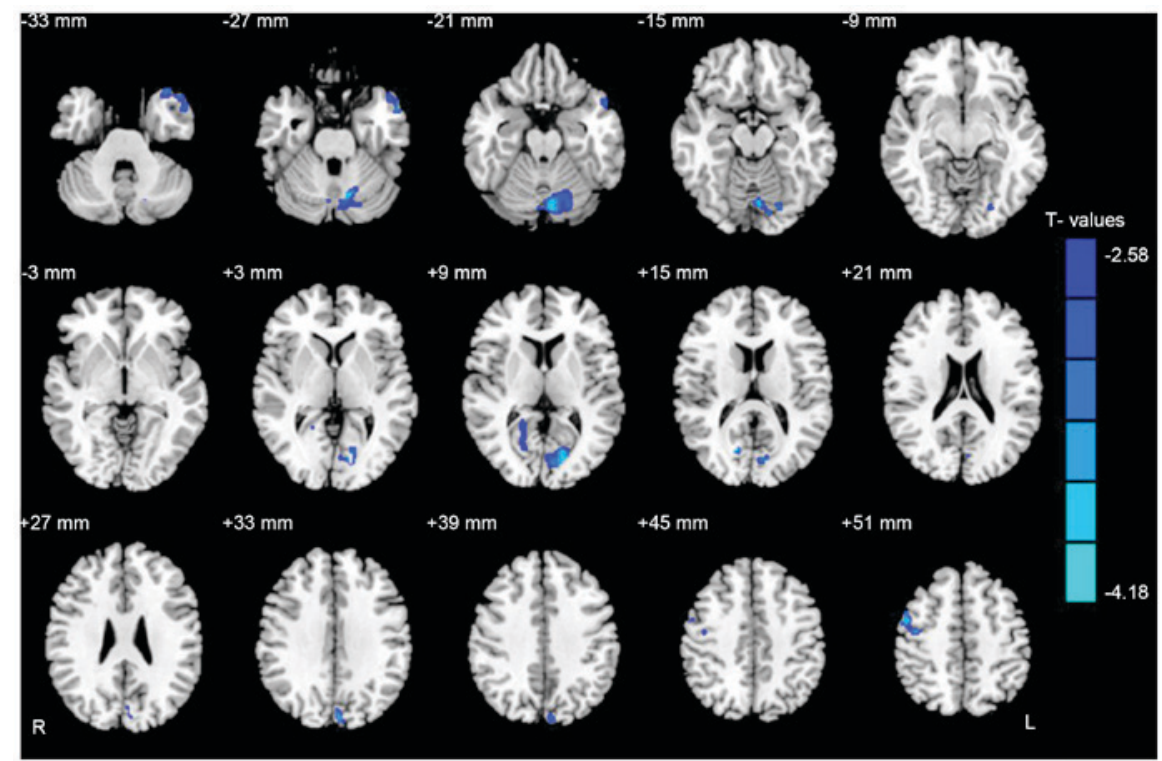

Figure 1. Regional GMV decrease in patients with SC compared with HCs. The significantly decreased regions were located in the left middle temporal pole, left cerebellum posterior lobe, right posterior cingulate cortex, left cuneus and right premotor cortex. The blue areas denote lower GMV brain regions ( $\mathrm{P}<0.05$ for multiple comparisons using Gaussian Random Field theory; voxels-wise $\mathrm{P}<0.01$ and cluster-wise $\mathrm{P}<0.05$ corrected). GMV, grey matter volume; $\mathrm{SC}$, comitant strabismus; HCs, healthy controls.

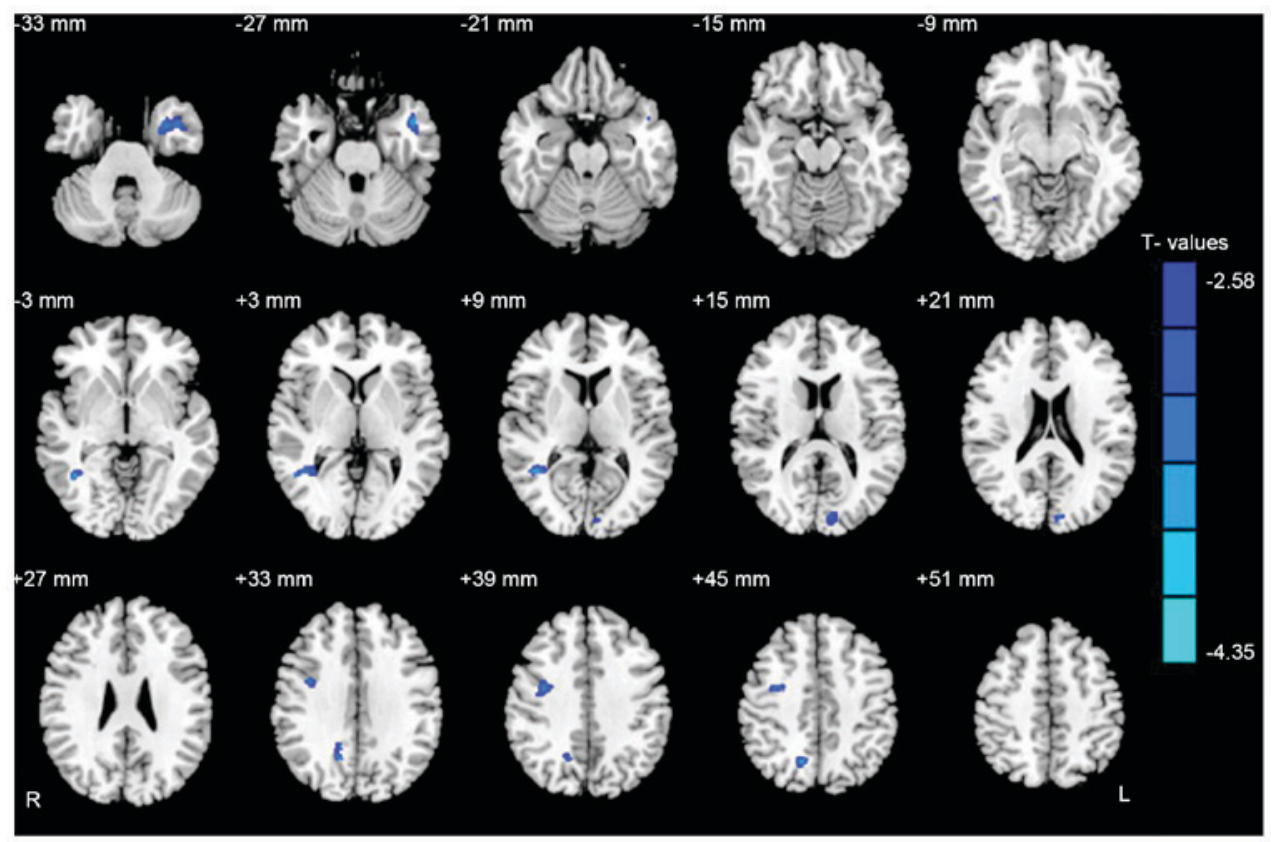

Figure 2. Regional WMV decrease in patients with CS compared with HCs. The significantly decreased regions were located in the left middle temporal gyrus, right middle temporal gyrus, right precuneus and right premotor cortex. The blue areas denote lower WMV brain regions $(\mathrm{P}<0.05$ for multiple comparisons using Gaussian Random Field theory; voxels-wise $\mathrm{P}<0.01$ and cluster-wise $\mathrm{P}<0.05$ corrected). WMV, white matter volume; SC, strabismus; HCs, healthy controls.

retinal image velocities (13). A previous study reported that the middle temporal gyrus contains a rudimentary representation of three-dimensional surface orientation (14). Additionally, the middle temporal gyrus has been suggested to encode three-dimensional motion (15). It has been well known that patients with CS often are accompanied with dysfunction of fusion and stereopsis (16). Yan et al (17) observed that patients with there had decreased WMV in the right inferior temporal gyrus in comitant exotropia. Duan et al (18) found that strabismic amblyopia indeed affected mean diffusion, not only in the occipital tracts but also the association tracts connecting the visual cortex to the frontal and temporal lobes. In agreement with these findings, the authors identified significantly decreased GMV values in the brain regions of the left middle temporal pole. Furthermore, the mean area of the GMV of the left middle temporal pole was 0.795 . There were significantly reduced WMV values in the bilateral middle temporal gyrus in the patients with CS, whereas that 
A

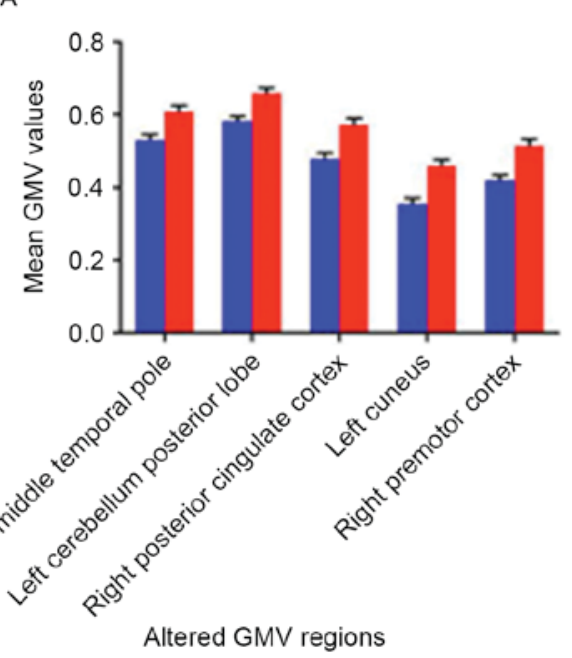

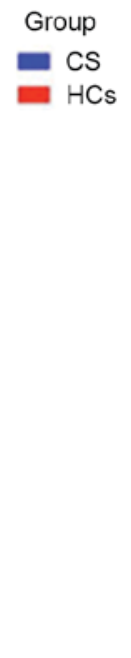

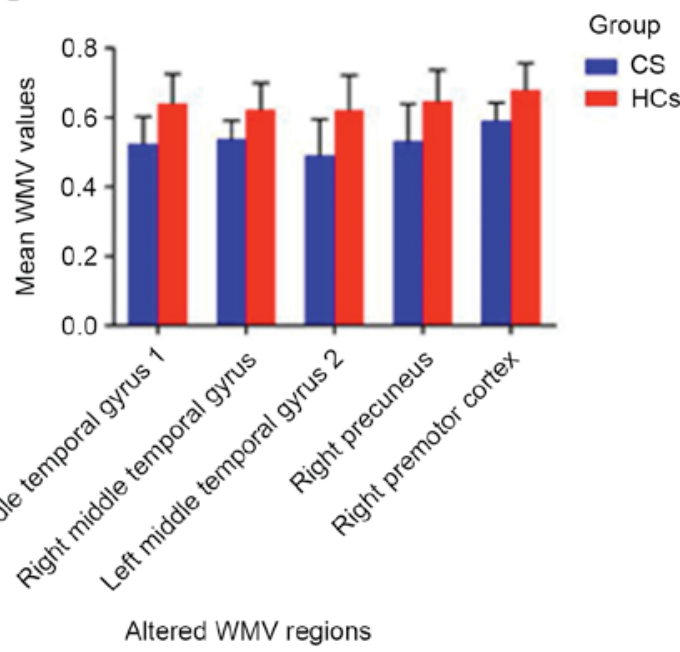

Figure 3. The mean of altered (A) GMV values and (B) WMV values between the CS group and the HCs group. Data are presented as the mean \pm standard deviation. GMV, grey matter volume; WMV, white matter volume; CS, comitant strabismus; HCs, healthy controls.

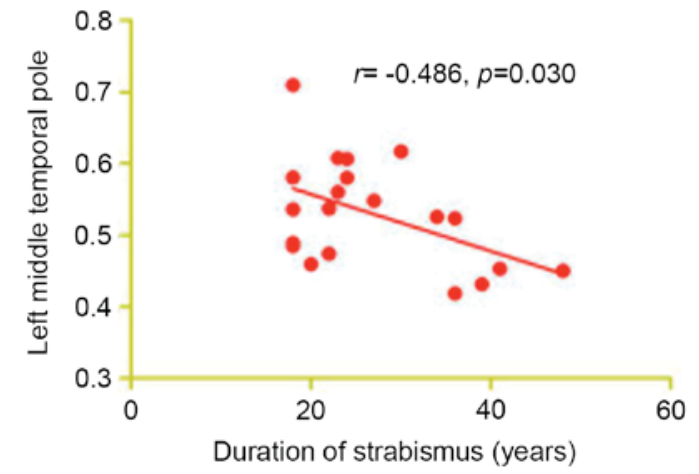

Figure 4. The correlations between the mean GMV values of different brain regions and the behavioral performances. The duration of strabismus negatively correlated with the GMV values of the left middle temporal pole $(r=-0.486, \mathrm{P}=0.030)$. GMV, grey matter volume.

of the WMV of the left middle temporal gyrus 1 was 0.810 , the right middle temporal gyrus was 0.818 and the left middle temporal gyrus 2 was 0.823 . It was demonstrated that there was both white matter and gray matter atrophy in CS patients. Therefore, the authors speculated that the CS possibly led to the atrophy of the temporal gyrus, which may reflect the impairment of the visual fusion in CS patients. Furthermore, the duration of CS presented a negative correlation with the GMV values of the left middle temporal pole $(r=-0.486$, $\mathrm{P}=0.030$ ). This suggests that a significant atrophy of the left middle temporal pole occur in the lingual gyrus during the early phase in CS.

The cerebellum is involved in the execution of accurate eye movements (19). A previous study reported that the cerebellum is responsible for the execution of eye movements (20). Other research reported that cerebellar vermis activation is related to visually guided saccades (21). Joshi et al (22) reported that the posterior interposed nucleus in the cerebellum is involved in eye movements in strabismic monkeys (22). Consistent with these findings, CS patients were observed to have significantly decreased GMV in the left cerebellum posterior lobe. The area
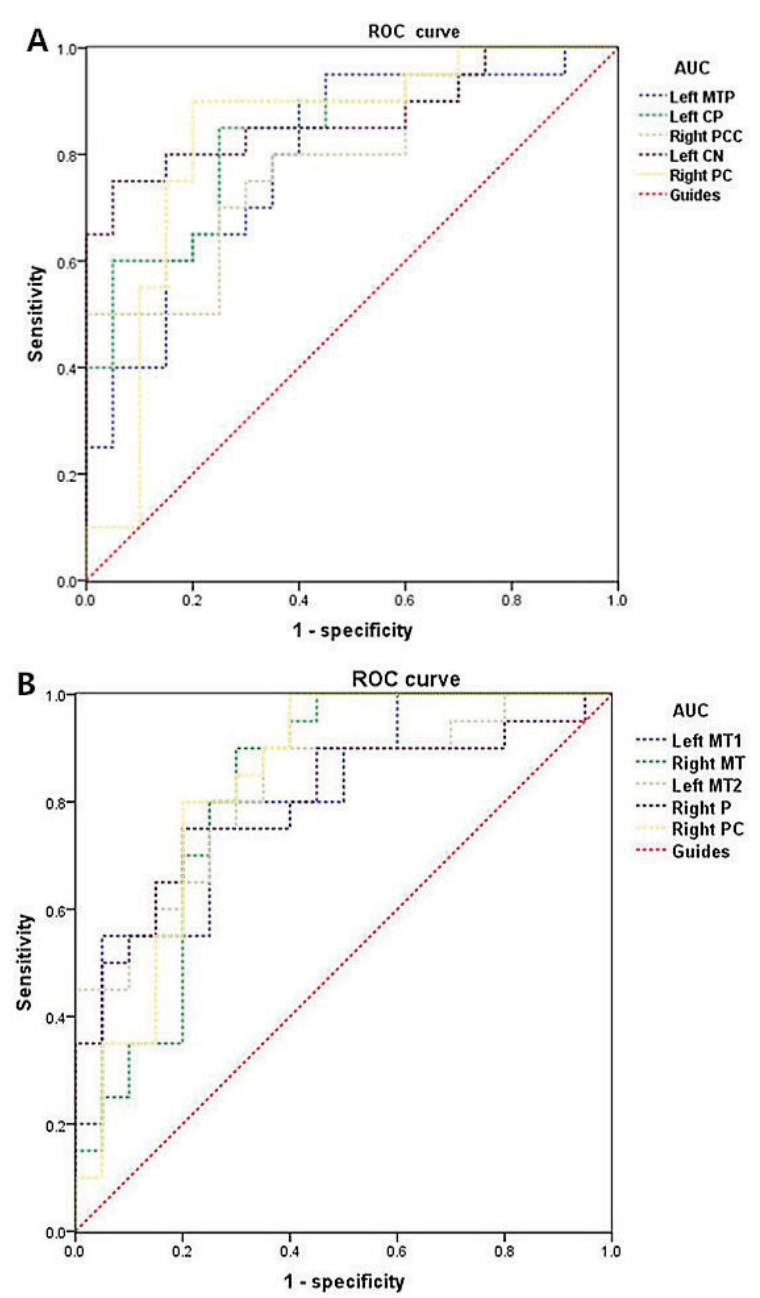

Figure 5. ROC curve analysis of the mean GMV and WMV values for altered brain regions. Note: (A) The areas under the ROC curve (AUCs) for GMV values: Left MTP, 0.795; left CP, 0.835; right PCC, 0.790; left CN, 0.870; right PC, 0.830. (B) The areas under the ROC curve (AUCs) for WMV values were: Left MT1, 0.810; right MT, 0.818; left MT2, 0.823; right $\mathrm{P}, 0.800$; right $\mathrm{PC}, 0.835$. ROC, receiver operating characteristic; GMV, grey matter volume; WMV, white matter volume; MTP, middle temporal pole; CP, cerebellum posterior lobe; PCC, posterior cingulate cortex; $\mathrm{CN}$, cuneus; PC, premotor cortex; MT, middle temporal gyrus; P, precuneus. 
under ROC curves of GMV of the left cerebellum posterior lobe was 0.835 . The authors therefore concluded that the CS may associate with deficits in the left cerebellum posterior lobe, reflecting eye movement damages.

Primary motor cortex, located in the primate frontal cortex (23), has been strongly implicated in motor processing (24). Previous studies have demonstrated the relationship between premotor cortex and the ocular movement $(25,26)$. As reported, the FEF is located in the posterior part of the middle frontal gyrus (27). A previous study demonstrated that the FEF is involved in the saccade selection and execution (28). Additionally, the FEF was also involved in sustained attention (29). Yan et al (17) found that WMV values were reduced in the right frontal lobe/sub-gyral in patients with comitant exotropia. In support of these findings, the authors also reported a significantly decreased WMV in the brain regions of the premotor cortex in CS patients. Furthermore, the CS patients presented a significantly decreased GMV in the regions of the premotor cortex. They came to the conclusion that the CS may lead to the atrophy of the premotor cortex, which may reflect the impaired oculomotor in the CS patients.

The precuneus is located in the rear region between the somatosensory cortex and forward of the cuneus between the two cerebral hemispheres. The precuneus is not only involved in the interwoven network of the self-consciousness (30), but also involved in different aspects of visuospatial mental operations (31). A previous study suggested that the bold signal was increased in the left cingulate gyrus, bilateral precuneus and left angular gyrus in the patients with infantile esotropia (32). In the present study, significantly decreased GMV values were observed in the brain regions of the left cuneus in CS patients. Meanwhile, CS patients had decreased WMV values in the brain regions of the right precuneus. The authors speculated that the CS may lead to the dysfunction of the right precuneus and the left cuneus.

The present study indicated that CS patients had decreased GMV and WMV in the different regions of the brain, which may give much important information to explain the underlying neural mechanisms of the fusion defects and ocular motility disorders in CS patients.

\section{Acknowledgements}

The present study was supported by the National Natural Science Foundation of China (grant nos. 81160118, 81460092 and 81400372), the Jiangxi Province Voyage Project (grant no. 2014022), the Natural Science Key Project of Jiangxi Province (grant no. 20161ACB21017), the Youth Science Foundation of Jiangxi Province (grant no. 20151BAB215016), the Technology and Science Foundation of Jiangxi Province (grant no. 20151BBG70223).

\section{References}

1. Adams DL, Economides JR and Horton JC: Contrasting effects of strabismic amblyopia on metabolic activity in superficial and deep layers of striate cortex. J Neurophysiol 113: 3337-3344, 2015.

2. Louwagie CR, Diehl NN, Greenberg AE and Mohney BG: Is the incidence of infantile esotropia declining?: A population-based study from Olmsted County, Minnesota, 1965 to 1994. Arch Ophthalmol 127: 200-203, 2009.
3. Koc F, Erten Y and Yurdakul NS: Does restoration of binocular vision make any difference in the quality of life in adult strabismus. Br J Ophthalmol 97: 1425-1430, 2013.

4. Iordanous Y, Mao A and Makar I: Preoperative factors affecting stereopsis after surgical alignment of acquired partially accommodative esotropia. Strabismus 23: 151-158, 2015.

5. Bi H, Zhang B, Tao X, Harwerth RS, Smith EL 3rd and Chino YM: Neuronal responses in visual area V2 (V2) of macaque monkeys with strabismic amblyopia. Cereb Cortex 21: 2033-2045, 2011.

6. Duan Y, Norcia AM, Yeatman JD and Mezer A: The Structural properties of major white matter tracts in strabismic amblyopia. Invest Ophthalmol Vis Sci 56: 5152-5160, 2015.

7. Chen VJ and Tarczy-Hornoch K: Functional magnetic resonance imaging of binocular interactions in visual cortex in strabismus. J Pediatr Ophthalmol Strabismus 48: 366-374, 2011.

8. Ashburner $\mathrm{J}$ and Friston KJ: Voxel-based morphometry-the methods. Neuroimage 11: 805-821, 2000.

9. Shimoda K, Kimura M, Yokota M and Okubo Y: Comparison of regional gray matter volume abnormalities in Alzheimer's disease and late life depression with hippocampal atrophy using VSRAD analysis: A voxel-based morphometry study. Psychiatry Res 232: 71-75, 2015.

10. Huang X, Zhang Q, Hu PH, Zhong YL, Zhang Y, Wei R, Xu TT and Shao Y; Oculopathy fMRI study group: White and gray matter volume changes and correlation with visual evoked potential in patients with optic Neuritis: A voxel-based morphometry study. Med Sci Monit 22: 1115-1123, 2016.

11. Kim GW and Jeong GW: White matter volume change and its correlation with symptom severity in patients with schizophrenia: A VBM-DARTEL study. Neuroreport 26: 1095-1100, 2015.

12. Chan ST, Tang KW, Lam KC, Chan LK, Mendola JD and Kwong KK: Neuroanatomy of adult strabismus: A voxel-based morphometric analysis of magnetic resonance structural scans. Neuroimage 22: 986-994, 2004.

13. Nguyenkim JD and DeAngelis GC: Disparity-based coding of three-dimensional surface orientation by macaque middle temporal neurons. J Neurosci 23: 7117-7128, 2003.

14. Sanada TM, Nguyenkim JD and Deangelis GC: Representation of 3-D surface orientation by velocity and disparity gradient cues in area MT. J Neurophysiol 107: 2109-2122, 2012.

15. Czuba TB, Huk AC, Cormack LK and Kohn A: Area MT encodes three-dimensional motion. J Neurosci 34: 15522-15533, 2014.

16. Feng X, Zhang X and Jia Y: Improvement in fusion and stereopsis following surgery for intermittent exotropia. J Pediatr Ophthalmol Strabismus 52: 52-57, 2015.

17. Yan X, Lin X, Wang Q, Zhang Y, Chen Y, Song S and Jiang T: Dorsal visual pathway changes in patients with comitant extropia. PLoS One 5: e10931, 2010.

18. Duan Y, Norcia AM, Yeatman JD and Mezer A: The structural properties of major white matter tracts in strabismic amblyopia. Invest Ophthalmol Vis Sci 56: 5152-5160, 2015.

19. Herzfeld DJ, Kojima Y, Soetedjo R and Shadmehr R: Encoding of action by the purkinje cells of the cerebellum. Nature 526: 439-442, 2015.

20. Nitschke MF, Arp T, Stavrou G, Erdmann C and Heide W: The cerebellum in the cerebro-cerebellar network for the control of eye and hand movements-an fMRI study. Prog Brain Res 148: 151-164, 2005.

21. Hayakawa Y, Nakajima T, Takagi M, Fukuhara N and Abe H: Human cerebellar activation in relation to saccadic eye movements: A functional magnetic resonance imaging study. Ophthalmologica 216: 399-405, 2002.

22. Joshi AC and Das VE: Muscimol inactivation of caudal fastigial nucleus and posterior interposed nucleus in monkeys with strabismus. J Neurophysiol 110: 1882-1891, 2013.

23. Boussaoud D and Wise SP: Primate frontal cortex: Neuronal activity following attentional versus intentional cues. Exp Brain Res 95: 15-27, 1993.

24. Shen L and Alexander GE: Neural correlates of a spatial sensory-to-motor transformation in primary motor cortex. J Neurophysiol 77: 1171-1194, 1997.

25. Halsband U, Matsuzaka Y and Tanji J: Neuronal activity in the primate supplementary, pre-supplementary and premotor cortex during externally and internally instructed sequential movements. Neurosci Res 20: 149-155, 1994.

26. Boussaoud D: Primate premotor cortex: Modulation of preparatory neuronal activity by gaze angle. J Neurophysiol 73: 886-890, 1995. 
27. Blanke O, Spinelli L, Thut G, Michel CM, Perrig S, Landis T and Seeck M: Location of the human frontal eye field as defined by electrical cortical stimulation: Anatomical, functional and electrophysiological characteristics. Neuroreport 11: 1907-1913, 2000.

28. Fernandes HL, Stevenson IH, Phillips AN, Segraves MA and Kording KP: Saliency and saccade encoding in the frontal eye field during natural scene search. Cereb Cortex 24: 3232-3245, 2014.

29. Esterman M, Liu G, Okabe H, Reagan A, Thai M and DeGutis J: Frontal eye field involvement in sustaining visual attention: Evidence from transcranial magnetic stimulation. Neuroimage 111: 542-548, 2015.
30. Cavanna AE and Trimble MR: The precuneus: A review of its functional anatomy and behavioural correlates. Brain 129: 564-583, 2006.

31. Oshio R, Tanaka S, Sadato N, Sokabe M, Hanakawa T and Honda M: Differential effect of double-pulse TMS applied to dorsal premotor cortex and precuneus during internal operationof visuospatial information. Neuroimage 49: 1108-1115, 2010.

32. Yang X, Zhang J, Lang L, Gong Q and Liu L: Assessment of cortical dysfunction in infantile esotropia using fMRI. Eur J Ophthalmol 24: 409-416, 2014. 